\title{
MRI findings in people with epilepsy and nodding syndrome in an area endemic for onchocerciasis: an observational study
}

\author{
*Winkler AS ${ }^{1,2}$, Friedrich $\mathrm{K}^{3}$, Velicheti $\mathrm{S}^{4}$, Dharsee $\mathrm{J}^{4}$, König $\mathrm{R}^{3}$, Nassri A ${ }^{3}$, Meindl $\mathrm{M}^{3}$, Kidunda $\mathrm{A}^{5}$, Müller \\ $\mathrm{TH}^{6}$, Jilek-Aall L7 , Matuja $\mathrm{W}^{8}$, Gotwald $\mathrm{T}^{9}$, Schmutzhard $\mathrm{E}^{3}$
}

1. Department of Neurology, Technical University of Munich, Germany

2. Haydom Lutheran Hospital, Manyara Region, Tanzania

3. Department of Neurology, Medical University of Innsbruck, Innsbruck, Austria

4. Department of Radiology, The Aga Khan Hospital, Dar es Salaam, Tanzania

5. Mahenge District Hospital, Morogoro Region, Tanzania

6. Institute for Medical Informatics, Biometry and Epidemiolgy, Ludwig-Maximilians-University, Munich, Germany

7. Department of Psychiatry, University of British Columbia, Vancouver, Canada

8. Department of Neurology, Muhimbili University of Health and Allied Sciences, Dar es Salaam, Tanzania

9. Department of Radiology, Medical University of Innsbruck, Innsbruck, Austria

\begin{abstract}
Background: Onchocerciasis has been implicated in the pathogenesis of epilepsy. The debate on a potential causal relationship between Onchocerca volvulus and epilepsy has taken a new direction in the light of the most recent epidemic of nodding syndrome.

Objective: To document MRI changes in people with different types of epilepsy and investigate whether there is an association with $O$. volvulus infection.

Methods: In a prospective study in southern Tanzania, an area endemic for O. volvulus with a high prevalence of epilepsy and nodding syndrome, we performed MRI on 32 people with epilepsy, 12 of which suffered from nodding syndrome. Polymerase chain reaction (PCR) of O. volvulus was performed in skin and CSF.

Results: The most frequent abnormalities seen on MRI was atrophy (twelve patients $(37.5 \%)$ ) followed by intraparenchymal pathologies such as changes in the hippocampus (nine patients $(28.1 \%)$ ), gliotic lesions (six patients $(18.8 \%)$ ) and subcortical signal abnormalities (three patients $(9.4 \%)$ ). There was an overall trend towards an association of intraparenchymal cerebral pathologies and infection with O. volvulus based on skin PCR (Fisher's Exact Test $\mathrm{p}=0.067$ ) which was most pronounced in children and adolescents with nodding syndrome compared to those with other types of epilepsy (Fisher's Exact Test, $\mathrm{p}=0.083)$. Contrary to skin PCR results, PCR of CSF was negative in all patients.

Conclusion: The observed trend towards an association of intraparenchymal cerebral pathological results on MRI and a positive skin PCR for O. volvulus despite negative PCR of CSF is intriguing and deserves further attention.

Key words: Epilepsy, head nodding, magnetic resonance imaging, cerebrospinal fluid. Onchocerca volvulus

African Health Sciences 2013; 13(2): 529 - 540 http://dx.doi.org/10.4314/ahs.v13i2.51
\end{abstract}

\section{Introduction}

The discussion whether onchocerciasis causes epilepsy still remains to be resolved and lately has taken a new direction in search for causes of a devastating African epidemic epilepsy syndrome termed nodding syndrome (NS). ${ }^{1-4}$ Human onchocerciasis is caused by the filarial nematode Onchocerca volvulus (O. volvulus), which mainly manifests

\section{*Corresponding author: \\ Dr. Andrea Sylivia Winkler \\ Department of Neurology \\ Klinikum rechts der Isar \\ Ismaninger Strasse 22, 81675 Munich \\ Germany \\ Tel.: +49/89/45815015 \\ Email: drawinkler@yahoo.com.au}

on skin and eyes. ${ }^{5,6}$ The blackfly (Simulium species) transmits the microfilariae $(\mathrm{mf})$ during a blood meal. In the host they grow into adult worms, which again mate and produce $\mathrm{mf}$. These $\mathrm{mf}$ mainly migrate in subcutaneous tissue and may occasionally be found in sputum, urine, blood, and cerebrospinal fluid (CSF). ${ }^{7,8}$ In African areas affected by onchocerciasis, some studies suggest a connection with epilepsy, ${ }^{9-14}$ while others do not. ${ }^{15-17}$ Overall a link between the two diseases seems possible, ${ }^{18,19}$ but is far from being established. Latest results on NS from South Sudan indicate that in some villages significantly more children with NS are infested with $O$. volvulus compared to healthy controls, whereas in other villages no difference could be found between cases and controls. ${ }^{3}$ 
One hypothesis is that $\mathrm{mf}$ may cause inflammation or direct destruction of brain tissue similar to the mechanisms known from the analysis of affected skin and eyes ${ }^{20}$. Therefore imaging studies may shed some more light on the debate whether there is a causal relationship between epilepsy and O. volvulus. The area (Mahenge) of southern Tanzania where the study took place has a prevalence of epilepsy of up to $37.1 / 1,000,{ }^{21}$ the prevalence rates of epilepsy in sub-Saharan Africa ranging from 7.8-14.8/1,000, ${ }^{22}$ and in addition, is highly endemic for onchocerciasis. ${ }^{23}$ Thus, it was deemed appropriate to recruit people with epilepsy (PWE) from this region for parasitological and neuroradiological work-up. To elucidate such a potential association between $O$. volvulus and epilepsy, we performed, for the first time, both cerebral MRI in PWE and children/adolescents with NS from an area endemic for O. volvulus together with O. volvulus polymerase chain reaction (PCR) of skin and CSF.

\section{Methods}

\section{Study sites and performance of MRI}

The main study was conducted in The Mahenge Epilepsy Clinic, which was founded in 1960 by L. JilekAall, nowadays attended by well over 900 people with epilepsy. ${ }^{24}$ It is situated in the Government Hospital of Mahenge which cares for an approximate 32,000 inhabitants of the Vigoi division, Ulanga district, southern Tanzania. Although Mahenge, our study area, has been part of the African Programme for Onchocerciasis Control (APOC) since 1997, in 2011 an epidemiological survey revealed that the prevalence of onchocerciasis was still 46\% (personal communication Elibariki Mwakapeje, Ministry of Health Tanzania).

The MRI scans were performed in the Department of Radiology at The Aga Khan Hospital, Dar es Salaam, $500 \mathrm{~km}$ away from Mahenge, using a 1.5 Tesla MR machine (GE). In all patients, axial T1W1, T2W1, FLAIR, coronal FLAIR, and sagittal T1W1 were performed. In selected patients (especially those patients with complex partial seizures or head nodding seizures), coronal T2W1 and coronal 3D SPGR for hippocampus anatomy was added. All scans were evaluated by three experienced radiologists (JD, SV and TG), who were blinded to the patient groups.

\section{Participants and data collection}

A convenience sample of patients known to suffer from epilepsy or NS was recruited from The Mahenge Epilepsy Clinic in order to evaluate whether in these people brain lesions on MRI were associated with a positive evidence for onchocerciasis. All PWE or NS had active epilepsy with at least one seizure in the year preceding the survey. Patients with single epileptic seizures or epileptic seizures with an obvious predisposition or cause (e.g. perinatal brain damage, seizures in the context of malaria or alcohol withdrawal seizures among others) were excluded. Epilepsy was defined as two or more afebrile seizures unrelated to acute metabolic disorders or withdrawal of drugs or alcohol. ${ }^{25}$ For the definition of NS refer to Winkler et al.. ${ }^{1}$ The actual head nodding seizure represents a repetitive short loss of neck muscle tone resulting in a forward bobbing of the head unexplained by any other neurological or psychiatric condition. In addition to the actual nodding seizures there may be other seizure types and/or neurological/medical signs/ symptoms present which has been suggested as early as 2008 to be termed nodding syndrome (=NS). ${ }^{1}$

A previously validated questionnaire was used in a face-to-face interview performed by neurologists (WM, ES, ASW), final-year medical students on elective attachment from the University of Innsbruck (KF, MM), a translator, at least one relative and the patient. Physical examination with neurological evaluation was also performed (WM, ES, ASW). An electroencephalogram (EEG) was not at hand. In terms of recruitment of participants we aimed for an even distribution of people with seemingly epilepsy without focal signs (group1) and epilepsy with focal signs (group 2), and within these two groups we aimed for similar ages, a balanced distribution of gender and people with $O$. volvulus positive and negative skin snips. Patients of group 1 suffered from generalized tonicclonic seizures and were neurologically and mentally normal. People with epileptic seizures (either focal or generalized based on ictal observation) with clear focal neurological signs and/or clear mental handicap/ cognitive impairment having been present before the onset of seizures were put into group 2. Alternatively, if on neurological and standard cognitive examination no abnormality was detected, patients with clear focal types of epilepsy but otherwise healthy were also put into this group. Patients with NS were allocated to a separate group (group 3) and divided into "head nodding only" and "head nodding plus" in order to reflect the full spectrum of disease $e^{1}$. The groups are summarized as follows:

\section{Patient groups}

Group 1 ( $n=10)$ : Epilepsy without obvious focal neurological signs/mental retardation

Group $2(n=10)$ : Epilepsy with obvious focal neurological signs/mental retardation, or clear focal epilepsy (based on ictal observations) without any of the mentioned signs

Group 3 ( $n=12$ ): Head nodding seizures, divided in:

a)Head nodding only" with head nodding seizures only 
b) «Head nodding plus" with head nodding seizures and other seizure types mainly generalized tonic-clonic seizures.

\section{Laboratory procedures}

Skin snips were taken from the right and left iliac crest. If there were skin changes suggestive of $O$. volvulus, a skin snip was taken from that site too. Microscopic evaluation of $\mathrm{mf}$ was performed at 40x magnification one and four hours later. If no $\mathrm{mf}$ was seen, the well was checked again after 12 hours. The $\mathrm{mf}$ density per person was calculated. The O. volvulus PCR was performed after a modified version of that used by Zhang et al. ${ }^{26}$ Methods were optimized, quality controls and specificity tests with different parasites were performed to exclude cross reactivity. Gel electrophoresis was applied to visualize the amplification products. The PCR for O. volvulus in CSF was performed according to the method used for skin snips. Infestation with $O$. volvulus was established in the presence of a positive skin PCR. In four cases PCR was not performed. In these cases the results on skin snip were observed.

\section{Ethical clearance}

The study was cleared by the Ethics Committee of the Muhimbili University College of Health Sciences, University of Dar es Salaam. Oral informed consent was given by all patients allowing us to transcribe clinical data into the questionnaire. Written and witnessed informed consent was obtained from the patients or, in case of children or non-competent patients, their respective parents or next of kin for all procedures.

\section{Statistical analysis}

The data was analysed using SPSS 18.0 (SPSS Inc., Chicago, USA). The chi square-test and Fischer's Exact test were used to test the association between two categorical variables. The Mann-Whitney $U$ test was performed for continuous variables with a nonparametric distribution; the significance level was set at alpha $=0.05$. As this is a hypothesis generating investigation, no adjustment for multiple testing was applied. Furthermore, in view of the small group sizes, no multivariate analyses were carried out.

\section{Results}

Demographic, clinical and laboratory details

The median age of all 32 patients was 16 years (interquartile range (IQR): 14-21 years) with a male to female ratio of $1.3: 1$. Along with demographic, clinical and imaging details, the average densities of the $O$. volvulus $\mathrm{mf}$ in the skin and the results of the respective PCR are presented in tables 2 - 4. In three cases, no further counting of $\mathrm{mf}$ was available, thus only information as to whether the skin snip was positive or negative is given. The skin snips of $28 / 32$ patients were retested for $O$. volvulus antigen by PCR. Five patients had a positive skin PCR despite a negative skin snip and on the other hand, three patients had a negative skin PCR despite a positive skin snip. The CSF PCR, the results of which are not presented in the tables, was performed in all patients and was negative in all of them.

\section{Results of the MRI investigations}

Normal cerebral MRI scans were found in 10/32 patients (31.3\%). Table 1 gives an overview of the diagnosed cerebral lesions.

Table 1: Pathologies on MRI scans divided into unilateral and bilateral lesions

\begin{tabular}{llll}
\hline Pathologies & $\begin{array}{l}\text { Unilateral } \\
\text { lesions }\end{array}$ & $\begin{array}{c}\text { Bilateral } \\
\text { lesions }\end{array}$ & Total \\
\hline HC changes* & 3 & 6 & 9 \\
Gliotic lesions** & 4 & 2 & 6 \\
Signal abnormalities*** & 0 & 3 & 3 \\
Cyst & 2 & 0 & 2 \\
Other lesions*** & 1 & 1 & 2 \\
Total & 10 & 12 & 22 \\
Atrophy & N.A. & N.A. & 12 \\
No biopsies & - & - & 10 \\
\hline
\end{tabular}

MRI could show more than one pathology. HC - Hippocampus, N.A. - not applicable

*Gliotic lesions were defined as focal small $(<1 \mathrm{~cm})$ punctuate hyperintensities in T2W1, mainly located in the frontal lobe; ${ }^{* *} \mathrm{HC}$ changes were divided into $\mathrm{HC}$ sclerosis and suspected $\mathrm{HC}$ sclerosis, the latter only showing either HC signal change or volume loss; ${ }^{* * *}$ Signal abnormalities were defined as subcortical hyperintensities that did not fulfil the criteria for gliotic lesions

****Other lesions: focal cortical dysplasia, bilateral cystic lesions in the occipital lobe 
Of the 32 patients, nine patients (28.1\%) showed changes in the hippocampus, six of them bilaterally. Sclerosis was confirmed in only three patients, the others had either signal changes or loss of volume in the hippocampus. Clinically $3 / 9$ patients had complex partial or generalized tonic clonic seizures with focal signs, 4/9 had nodding seizures either alone or associated with other seizure types and one patient had generalized tonic clonic seizures without focal signs. Only three patients with hippocampus changes reported febrile seizures in childhood. Six patients $(18.8 \%)$ had gliotic lesions, mainly subcortically, and three patients (9.4\%) showed bilateral peritrigonal/ subcortical signal abnormalities.In two patients $(6.3 \%)$ an arachnoid cyst was found, one in the frontal lobe measuring 20x14 mm, the other in the temporal lobe being much smaller in size. A focal cortical dysplasia in the frontal lobe and a bilateral cystic lesion in the occipital lobes, the latter most likely caused by perinatal hypoxic brain damage, were seen in one patient each. Atrophy of the brain was found in $12 / 32$ patients $(37.5 \%)$ and was the only pathological finding in four of them. Cerebral and cerebellar atrophy were equally distributed (tables 1-4).

In group 1, four patients showed intracerebral pathologies on MRI. Unilateral gliotic lesions, focal cortical dysplasia and bilateral signal abnormalities were seen as well as unilateral hippocampus changes (table 2).

Table 2 : Demographics, neurological examination, MRI, mf density and skin PCR of group 1

\begin{tabular}{|c|c|c|c|c|c|c|c|}
\hline $\begin{array}{l}\text { Age } \\
\text { (yrs) }\end{array}$ & Gende & $\begin{array}{c}\text { er Seizure } \\
\text { type }\end{array}$ & $\begin{array}{l}\text { Neurological } \\
\text { examination/ } \\
\text { mental exami- } \\
\text { nation }\end{array}$ & $\begin{array}{l}\text { Intraparenchymal } \\
\text { MRI pathologies }\end{array}$ & Atrophy & Mf skin /mg & PCR skin \\
\hline 12 & M & Gen & NAD & Susp. HC sclerosis $1 \mathrm{ft}$ & No & 6 & Pos \\
\hline 17 & M & Gen & NAD & $\begin{array}{l}\text { Cortical dysplasia lft } \\
\text { frontal }\end{array}$ & No & 51.5 & Pos \\
\hline 20 & M & Gen & NAD & $\begin{array}{l}\text { Signal abnorm. bilateral } \\
\text { peritrigonal }\end{array}$ & General & 11.5 & Pos \\
\hline 21 & $\mathrm{~F}$ & Gen & NAD & $\begin{array}{l}\text { Gliotic lesions subcortical } \\
\text { rt frontal }\end{array}$ & No & 1.33 & Pos \\
\hline 35 & $\mathrm{~F}$ & Gen & NAD & No & No & Pos* & Pos \\
\hline 16 & $\mathrm{~F}$ & Gen & NAD & No & Mild general & 0 & Neg \\
\hline 18 & M & Gen & NAD & No & No & 0 & Pos \\
\hline 20 & $\mathrm{~F}$ & Gen & NAD & No & No & 0 & Neg \\
\hline 21 & M & Gen & NAD & No & No & 0 & $\mathrm{Neg}$ \\
\hline$\underline{32}$ & $\mathrm{M}$ & Gen & NAD & No & General & 0 & $\mathrm{Neg}$ \\
\hline
\end{tabular}

Gen - generalized tonic clonic seizures, NAD - nothing abnormal detected, rt - right, lft- left, susp. - suspected, abnorm. - abnormalities, Mf - microfilariae, pos* - no further counting available

In group 2, two patients had normal MRI, one without pathological findings on neurological and mental examination and the other with slight mental retardation. All other patients had pathological MRI results which together with the clinical examination, mf density and skin PCR are shown in table 3. The most frequent intraparenchymal cerebral pathologies were signal changes in the hippocampus followed by subcortical signal abnormalities (table 3 ).

In group 3, consisting of patients with NS, four patients $(33.3 \%)$ with head nodding alone showed normal MRI scans. The remaining eight patients, the majority of which suffered from NS together with generalized tonic clonic seizures, showed gliotic lesions (5/8; figures 1a-6a) and hippocampus pathologies (4/ $8)$, among other lesions (table 4).

\section{Relationship between results on MRI and types of epilepsy (subgroup analysis)}

The presence of intraparenchymal pathologies on MRI (exclusive of cerebral atrophy) was not dependent on the patients' age (Mann-Whitney $U$ test, $\mathrm{p}=0.985$ ) or gender $\left(c^{2}\right.$-test, $\left.p=0.530\right)$. Regarding types of epilepsy, relevant intraparenchymal MRI pathologies such as hippocampus changes, gliotic lesions and signal abnormalities, were significantly more frequent in the group "head nodding plus with other seizure types" compared to the other types of epilepsy (Fisher's Exact Test, $\mathrm{p}=0.01$; table 4). When looking at specific brain lesions, the significance could be maintained for gliotic lesions only (Fisher's Exact Test, $\mathrm{p}=0.034$; figures 1a6a). 
Table 3: Demographics, neurological examination, MRI, mf density and skin PCR of group 2

\begin{tabular}{|c|c|c|c|c|c|c|c|}
\hline $\begin{array}{l}\text { Age } \\
\text { (yrs) }\end{array}$ & Gende & $\begin{array}{l}\text { er Seizure } \\
\text { type }\end{array}$ & $\begin{array}{l}\text { Neurological examin- } \\
\text { ation/ mental examin- } \\
\text { ation }\end{array}$ & $\begin{array}{l}\text { Intraparenc- } \\
\text { hymal MRI path- } \\
\text { ologies }\end{array}$ & Atrophy & $\begin{array}{l}\text { Mf skin } \\
/ \mathrm{mg}\end{array}$ & $\begin{array}{l}\text { PCR } \\
\text { skin }\end{array}$ \\
\hline 14 & $\mathrm{~F}$ & $\begin{array}{l}\text { 1.Gen+, } \\
\text { 2.Cp }\end{array}$ & NAD & No & No & 0.5 & $\begin{array}{l}\text { Not } \\
\text { tested }\end{array}$ \\
\hline 16 & M & Gen & $\begin{array}{l}\text { MeRe, no focal neurol- } \\
\text { ogical signs }\end{array}$ & No & $\begin{array}{l}\text { Cerebellar } \\
\text { vermis }\end{array}$ & 4 & Neg \\
\hline 18 & $\mathrm{~F}$ & Gen & $\begin{array}{l}\text { MeRe, no focal neurol- } \\
\text { ogical signs }\end{array}$ & Arachnoid cyst rt frontal & No & 35.5 & Pos \\
\hline 22 & M & Gen+ & $\begin{array}{l}\text { MeRe,hemihypaesthesia } \\
\text { rt, UMN signs rt }\end{array}$ & Susp. HC sclerosis bilateral & $\begin{array}{l}\text { Mild } \\
\text { general }\end{array}$ & 0.17 & Pos \\
\hline 47 & M & $\begin{array}{l}\text { 1.Gen, } \\
\text { no focal } \\
\text { neurologica } \\
\text { signs }\end{array}$ & NAD & $\begin{array}{l}\text { HC sclerosis lft, susp. } \\
\text { HC sclerosis rt; signal } \\
\text { abnorm. subcortical bilateral } \\
\text { frontal }\end{array}$ & Cerebellar & 5.5 & Pos \\
\hline 13 & $\mathrm{~F}$ & $\mathrm{Cp}$ & $\begin{array}{l}\text { MeRe, no focal neurol- } \\
\text { ogical signs }\end{array}$ & No & No & 0 & Pos \\
\hline 13 & M & $\begin{array}{l}\text { 1.Gen, } \\
\text { 2.Cp }\end{array}$ & $\begin{array}{l}\text { MeRe, no focal neurol- } \\
\text { ogical signs }\end{array}$ & $\begin{array}{l}\text { Signal abnorm. subcortical } \\
\text { bilateral occipital }\end{array}$ & No & 0 & Neg \\
\hline 15 & M & Gen & MeRe, cerebellar syndrome & $\begin{array}{l}\text { Cystic defects occipital } \\
\text { bilateral }\end{array}$ & Cerebellar & 0 & Neg \\
\hline 16 & M & Gen & MeRe, increased reflexes & No & $\begin{array}{l}\text { Mild cere- } \\
\text { bellar }\end{array}$ & 0 & Neg \\
\hline 27 & $\mathrm{~F}$ & Gen+ & NAD & Susp. HC sclerosis bilateral & $\begin{array}{l}\text { Cerebral } \\
\text { occipital }+ \\
\text { parietal }\end{array}$ & 0 & Neg \\
\hline
\end{tabular}

Cp - complex partial seizures, Gen - generalized tonic clonic seizures, Gen+ - generalized tonic clonic seizures with additional focal signs ictally, NAD - nothing abnormal detected, MeRe - mental retardation, UMN - upper motor neuron, HC - Hippocampus, lft - left, rt - right, susp. - suspected, abnorm. - abnormalities, Mf - microfilariae, HC changes were divided into HC sclerosis and suspected HC sclerosis, the latter only showing either HC signal changes or volume loss.

Table 4: Demographics, neurological examination, MRI, mf density and skin PCR of group 3

\begin{tabular}{|c|c|c|c|c|c|c|c|}
\hline $\begin{array}{l}\text { Age } \\
\text { (yrs) }\end{array}$ & Gender & $\begin{array}{l}\text { Seizure } \\
\text { type }\end{array}$ & $\begin{array}{l}\text { Neurological examinat- } \\
\text { ion/mental examination }\end{array}$ & $\begin{array}{l}\text { Intraparenchymal } \\
\text { MRI pathologies }\end{array}$ & Atrophy & $\begin{array}{l}\text { Mf } \\
\text { skin / }\end{array}$ & $\begin{array}{l}\text { PCR skin } \\
\mathrm{g}\end{array}$ \\
\hline 15 & M & HN only & NAD & No & No & 1.5 & Neg \\
\hline 16 & $\mathrm{~F}$ & HN only & NAD & $\begin{array}{l}\text { Gliotic lesions rt frontal } \\
\text { subcortical; susp. HC } \\
\text { sclerosis rt }>\text { lft }\end{array}$ & No & 5.65 & Pos \\
\hline 18 & M & HN only & $\begin{array}{l}\text { Frontal release signs, } \\
\text { UMN signs lft }>\text { rt }\end{array}$ & No & No & 1 & Neg \\
\hline 10 & F & HN only & NAD & No & No & 0 & Not tested \\
\hline 14 & $\mathrm{~F}$ & HN only & NAD & $\mathrm{No}$ & No & Neg* & Not tested \\
\hline 15 & M & HN only & NAD & $\begin{array}{l}\text { Arachn cyst rt } \\
\text { temporal; HC sclerosis rt }\end{array}$ & No & 0 & Pos \\
\hline 9 & $\mathrm{~F}$ & HN only & UMN signs $\mathrm{rt}$ & $\begin{array}{l}\text { Gliotic lesions rt frontal } \\
\text { subcortical; susp. HC } \\
\text { sclerosis rt }>\text { lft }\end{array}$ & General & Pos* & Not tested \\
\hline 15 & M & $\begin{array}{l}\text { 1.HN, } \\
\text { 2.Gen }\end{array}$ & NAD & $\begin{array}{l}\text { Gliotic lesions bilateral } \\
\text { lft }>\text { rt frontal subcortical }\end{array}$ & No & 1.5 & Pos \\
\hline 16 & $\mathrm{~F}$ & $\begin{array}{l}\text { 1. } \mathrm{HN}, \\
\text { 2.Gen }\end{array}$ & NAD & $\begin{array}{l}\text { Gliotic lesions lft temp- } \\
\text { oral-occipital-parietal } \\
\text { gwm-j }\end{array}$ & No & 6.17 & Pos \\
\hline 13 & M & $\begin{array}{l}\text { 1.HN, } \\
\text { 2.Gen }\end{array}$ & NAD & $\begin{array}{l}\text { Gliotic lesions bilateral } \\
\text { frontal subcortical }\end{array}$ & No & 0 & $\mathrm{Neg}$ \\
\hline 16 & F & $\begin{array}{l}\text { 1.HN, } \\
\text { 2.Gen }\end{array}$ & NAD & $\begin{array}{l}\text { Susp. HC sclerosis lft } \\
\text { Mild cerebellar }\end{array}$ & 0 & Pos & \\
\hline 21 & M & $\begin{array}{l}\text { 1.HN, } \\
\text { 2.Gen, } \\
\text { 3.Cp }\end{array}$ & $\begin{array}{l}\text { Brain damage, no focal } \\
\text { neurology }\end{array}$ & $\begin{array}{l}\text { Susp. HC sclerosis } \\
\text { bilateral }\end{array}$ & $\begin{array}{l}\text { Mild } \\
\text { cerebellar }\end{array}$ & 0 & Pos \\
\hline
\end{tabular}


Footnotes for table 4

$\mathrm{HN}$ - head nodding seizures, Gen - generalized tonic clonic seizures, Cp - complex partial seizures, NAD nothing abnormal detected, HC - Hippocampus, susp. - suspected, gwm- $\mathrm{j}$ - grey-white matter junction, $\mathrm{rt}$ right, lft - left, neg*, pos* - no further counting available

HC changes were divided into HC sclerosis and suspected HC sclerosis, the latter only showing either HC signal changes or volume loss.

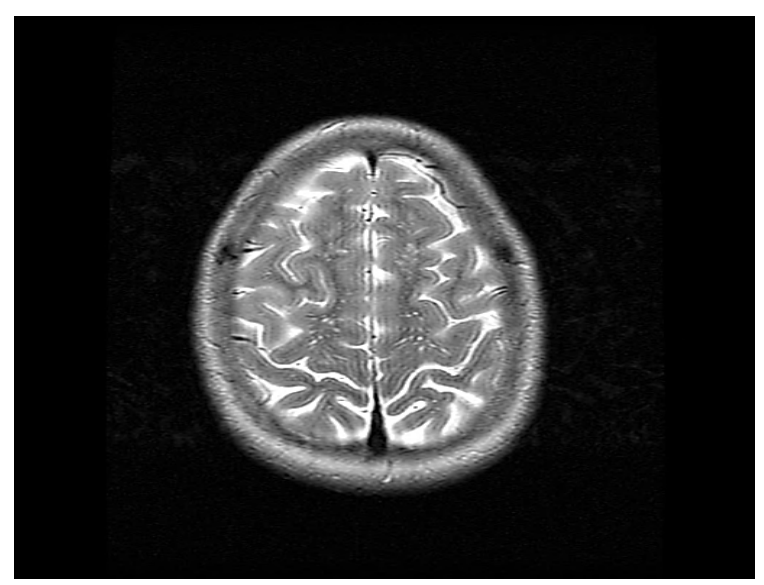

Figure 1a T2W1

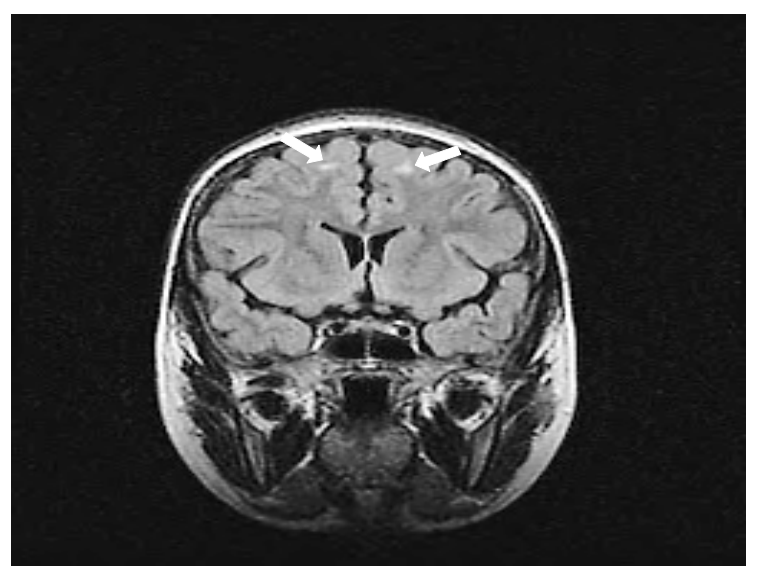

Figure 2a-b FLAIR

Figures 1a - 3a: 13 year-old male with head nodding and generalized tonic clonic seizures showing bilateral punctuate subcortical hyperintensities in the frontal lobe on T2W1. Some of them are suppressed on FLAIR confirming that they are Virchow-Robin spaces, the remaining hyperintense foci most likely correspond to gliotic lesions. After administration of contrast medium, lesions were non-enhancing on T1W1.

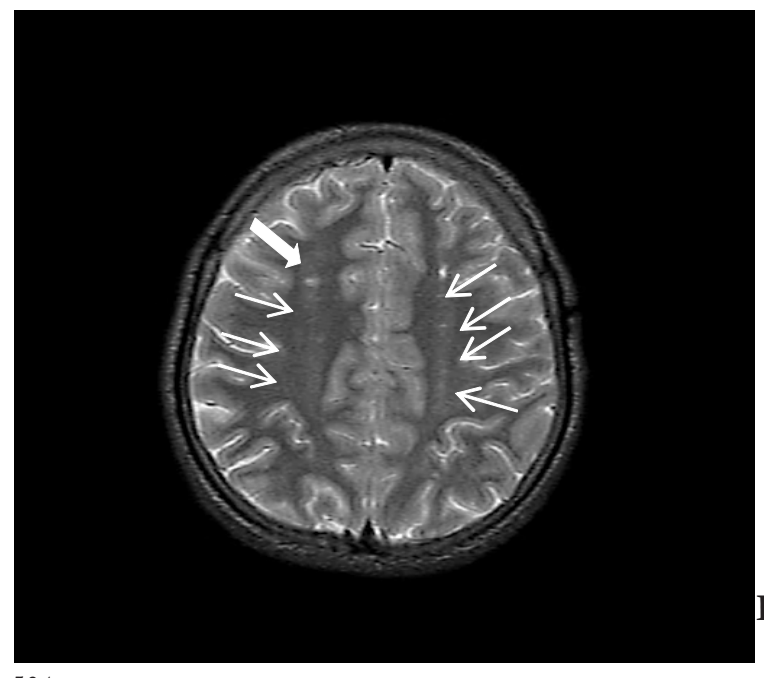

Figure 4a T2W1 


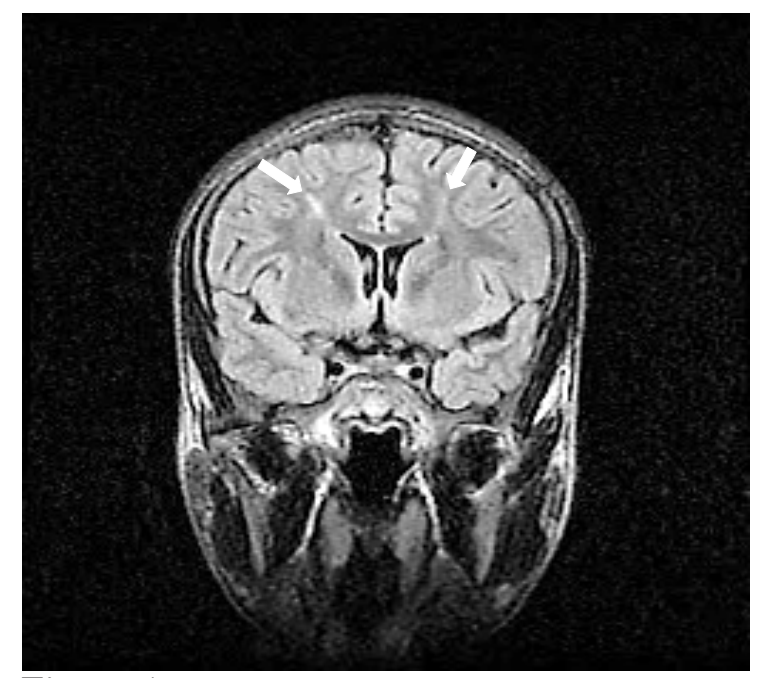

Figure 5a

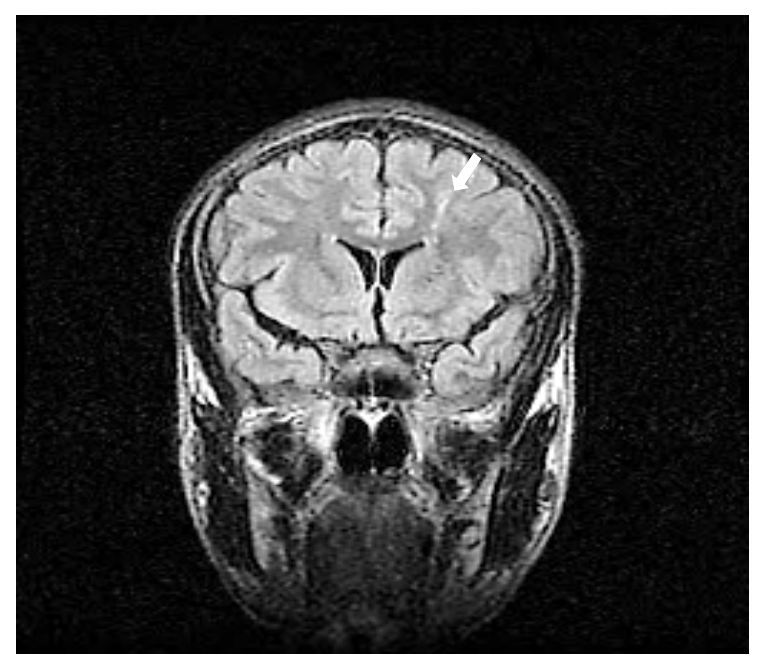

Figure $5 b$

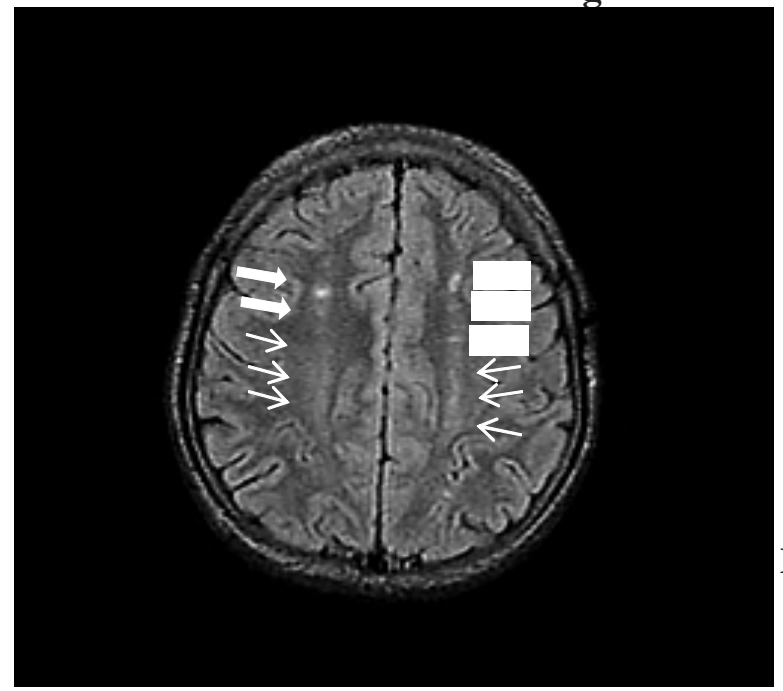

Figure 5c

Figure 5a-c FLAIR

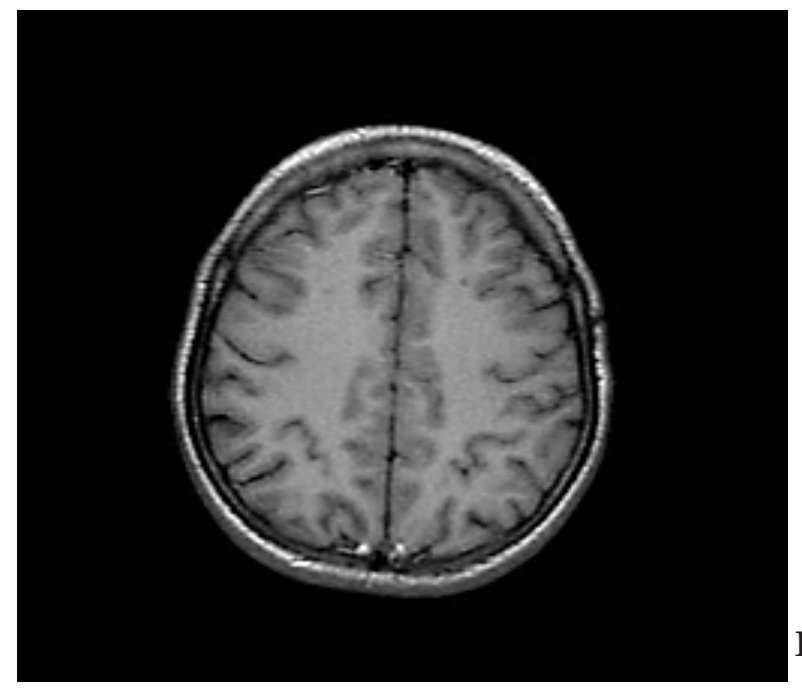

Figure 6a T1W1 without contrast

Figures 4a-6a: 14 year-old male with head nodding and generalized tonic clonic seizures showing bilateral punctuate subcortical hyperintensities in the frontal lobe, on T2W1 and axial as well as coronal FLAIR most likely corresponding to gliotic lesions 


\section{Relationship between results on MRI and infestation with $O$. volvulus}

Patients with an abnormal MRI had significantly more positive skin PCR results (Fisher's Exact Test $\mathrm{p}=0.019$ ) than patients with a normal MRI (exclusive of atrophy). When looking at plausible lesions that could be caused by O. volvulus including gliotic lesions, hippocampus changes or signal abnormalities there was a near significant trend for these lesions to be associated with a positive PCR results (Fisher's Exact Test $\mathrm{p}=0.067)$. Interestingly, when looking at epilepsy types, it was only in people with head nodding that the trend towards an association of intraparenchymal pathologies on MRI with a positive skin PCR for $O$. volvulus was seen (Fisher's Exact Test, $\mathrm{p}=0.083$; table 4).

When all three types of lesions were further analysed separately the association could not be maintained.

\section{Discussion}

\section{Our findings in general}

The International League Against Epilepsy has recommended neuroimaging with MRI for all PWE wherever feasible. ${ }^{27}$ Availability and affordability are rendering this recommendation almost impossible in resource-poor countries. Excluding South Africa, only nine MRI scanners were available in sub-Saharan countries in $2003 .{ }^{28} \mathrm{CT}$ scans are less expensive and more widely-spread; in 200365 CT were registered throughout sub-Saharan Africa. ${ }^{28}$ Thus, imaging studies in PWE in sub-SaharanAfrica are scarce and were mainly performed by means of CT. ${ }^{29,30}$ In our study, we performed 32 MRI scans in PWE with different types of epilepsies including children/ adolescents with NS with and without additional types of seizures. Parts of the findings in children/ adolescents with NS were already summarized in the description of the study population in 2008, figure 1 and are now presented more in depth and in the wider context of other types of epileptic seizures.

Only 10 (31\%) PWE of our study population showed no abnormality on MRI. This is highly different from studies from Brazil in which $76 \%$ of over 130 PWE had normal MRI scans figure 3 and from Nigeria in which almost 50\% of PWE had a normal CT scans. ${ }^{30}$ The reason for the high number of abnormalities in our study might be explained by selection bias. The patients of our study with normal MRI were mainly suffering from epilepsy without any obvious neurological signs (group 1), emphasizing the fact that thorough history taking associated with clinical examination is a good indicator for the type of epilepsy in resource-poor settings.

In the individuals with pathological MRI scans, atrophy, hippocampus changes and gliotic lesions were the commonest findings. In some patients, the atrophy was disproportionate for age. Atrophy of the cerebellum may be explained by high doses of phenytoin over many years and cerebral atrophy by excessive alcohol use. Changes in the hippocampus were seen in over $20 \%$ of PWE, which may cause complex partial seizures, including head nodding in children. ${ }^{32}$ Interestingly, all but one PWE with hippocampus changes in our study had seizures in the context of either focal epilepsy or NS. In our study, no significant relationship between febrile convulsions and hippocampus changes was found. Febrile seizures have however been identified as risk factors of childhood epilepsy in other African studies. ${ }^{33-35}$ Gliotic lesions were mainly found in children and adolescents with head nodding, especially in those with additional seizure types. Cranial infections, cerebral ischemia and traumatic brain injury have all been implicated as possible causes for gliotic lesions in a study from China where $60 \%$ of people with gliotic lesions were suffering from epilepsy. ${ }^{36}$ There was no history of trauma in the people of our study. Ischemia especially in younger people seems unlikely, so that an inflammatory cause for the gliotic lesions seems possible.

\section{MRI findings and $O$. volvulus}

Electroencephalographic studies in PWE from an area endemic for O. volvulus with high prevalence of epilepsy showed mainly focal epileptiform activity. ${ }^{37}$ Focal lesions/pathologies were also prevalent in our study population mainly consisting of gliotic lesions and hippocampus changes. They were not evenly distributed over the three study groups, but seemed to be clustering in PWE with clinical indicators pointing to focal epilepsy and in children/adolescents with NS, especially those suffering from NS and additional types of seizures. Hippocampus changes seem rather difficult to attribute to an infectious agent but have been implicated in the context of neurocysticercosis caused by the parasite Taenia solium, ${ }^{38}$ whereas gliotic changes may be attributed to inflammation. ${ }^{36} \mathrm{Mf}$ migrating into the brain may cause focal inflammatory reaction and subsequent gliosis as has been shown with Loa loa, a filarial nematode mainly distributed in West and Central Africa. L. loa adult worms have been found to be able to enter the brain causing an acute and severe 
encephalopathy mainly in O. volvulus co-infected individuals treated with filaricidal drugs. Occasional cases were reported, but are rare in comparison to the number of individuals affected by $L$. loa.$^{39}$ Pathological changes in L. loa encephalopathy include inflammatory infiltrates and gliotic lesions in brain parenchyma of various degrees of severity. ${ }^{40}$ In addition to direct damage to brain parenchyma affection of cerebral arteries (cellular infiltrates and thickening of the vessel wall) has also be seen observed $^{40}$ which may lead to potentially ensuing vasculitis that has also been demonstrated with other parasites such as Toxocara canis. ${ }^{41}$

Duke et al. ${ }^{8}$ reported O. volvulus $\mathrm{mf}$ in CSF before and after treatment with diethylcarbamazine. If this is a genuine finding and was not due to contamination during lumbar puncture, the CSF should have shown some signs of contact with the parasites. Unfortunately, this was not described in Duke's study. In our study, O. volvulus PCR of CSF was negative in all individuals. It may be argued that if the inflammation caused by $\mathrm{mf}$ had resolved, $O$. volvulus PCR, which traces DNA and thus an active process, may become negative. Interestingly, in patients of a much bigger patient cohort of almost 200 PWE of which PWE of the current study were part of, O. volvulus CSF antibody index, which should give evidence of exposure to the parasite, was negative too, indicating that contact of the CSF with the parasite, past or present, seems very unlikely. ${ }^{42}$

Gliotic lesions or other types of signal abnormalities may also be seen in an autoimmune response. Gallin et al. ${ }^{43}$ have demonstrated an association between a hyperreactive onchodermatitis and the presence of autoantibodies to proteins found in neutrophils in affected individuals. Also, an autoimmune process has been hypothesized in the development of ocular onchocerciasis and a shared antigen (OV39) between ocular tissue, including the retina, and O. volvulus has been implicated in the disease process. ${ }^{44,45}$ Based on these principles, cross-reacting antibodies to nervous tissue may be postulated. ${ }^{20,42}$ One would however expect a completely different, at least more widespread "gliotic" reaction if an autoimmune pathogenesis caused by $O$. volvulus is assumed.

An indirect relationship between $O$. volvulus and epilepsy might further be supported by the fact that we found an almost significant association between intraparenchymal pathologies on MRI and O. volvulus PCR result on skin snip. Also, when looking at the various types of epilepsy a nearly significant relationship between brain pathologies, especially gliosis and hippocampus changes, and positive skin PCR could be maintained for children/adolescents with NS only. Whether these lesions are causative for the nodding seizures and/or the additional mainly generalized seizures or whether they are unrelated remains uncertain in the absence of a control group. Interictal EEG results in some of these children showed generalized rather than focal abnormalities, ${ }^{1}$ which however does not exclude gliosis or hippocampus changes as origin. As to the actual head nodding attack, which clinically looks like atonic seizures (and has recently been shown to be reflected by an EEG decrement ${ }^{46}$ ) or, if nodding is less prominent, complex partial or absence-type seizures, gliosis as the cause of these attacks seem less likely and in fact only $2 / 7$ children with head nodding attacks alone had gliotic lesions on MRI (table 4). Hippocampus changes were seen in five of the 12 children/adolescents with NS and may cause complex partial seizures in this cohort. However, hippocampus changes were not more frequent than in PWE with focal seizures and therefore may be an accidental finding without implication for seizure generation in children/adolescents with NS or, alternatively, some of the children/adolescents with NS may indeed suffer from complex partial epilepsy which clinically appears as head nodding. ${ }^{32}$

\section{Limitations of the study}

This is more of a descriptive study of MRI pathologies in PWE and children/adolescents with NS from an O. volvulus endemic area. In this context statistical analysis of MRI pathologies and their relationship with onchocerciasis must be interpreted with care due to the small sample size. Local circumstances with the only MRI in the country being many hundreds of kilometers away and financial restrictions did not allow for more people to be recruited into the study. Also, the MRI (1.5 Tesla) used in our study shows a fairly low resolution compared to a 3 Tesla MRI and lesions may have been missed. Neuroimaging in a larger population, especially of children/adolescents with NS, should be envisaged in order to support or reject the trends observed in our study. Also, our study is lacking a control group of healthy age-matched individuals. Healthy young people in the circumstances of a resource-poor setting can usually not be awayfor several days as they are the backbone of their families and indeed all the young people approached by us did not consent to participate into our study. 


\section{Conclusion}

MRI in PWE and children/adolescents with HS from an area endemic for O. volvulus shows multiple pathologies, the most frequent being atrophy, hippocampus changes and gliosis. There was a trend towards an association of intraparenchymal brain pathologies (hippocampus changes, gliotic lesions as well as other signal abnormalities) and O. volvulus skin PCR in PWE and more explicitly in children/ adolescents with NS. However, as this was a mere observational study with low numbers and without control group, the possible association between certain brain pathologies and infection with $O$. volvulus should be explored further, preferably in larger studies with matched control groups.

\section{Abbreviations}

CSF: cerebrospinal fluid; L. Loa: Loa loa; NS: nodding syndrome; MRI: magnetic resonance imaging; $O$. volvulus: Onchocerca volvulus; PCR: polymerase chain reaction; PWE; people with epilepsy

\section{Acknowledgments}

The study was supported by the Savoy Epilepsy Foundation, Quebec, Canada and by a grant of the Department of Neurology, NICU, Medical University Innsbruck, Austria. ASW was supported by the Centre for International Migration (CIM), Frankfurt, Germany.

We are very grateful to the staff of: The Mahenge Epilepsy Clinic, The Mahenge District Hospital and Aga Kahn Hospital (especially Dr. Jaffer Dharsee and Dr. Sandeep Velicheti), Dar es Salaam, who were ever so helpful. We also want to thank the Home for Disabled People, Msimbazi, who always warmly welcomed our patients and gave them shelter and food. Our special thanks go to all the patients and their relatives, who participated in our study. We are also indebted to the members of the Department of Infectious Diseases and Tropical Medicine, Ludwig-Maximilians-University, Munich, for allowing us to use their facilities and for their input into performing the PCR. Special thanks go to Prof. Louise Jilek-Aall for her pioneering work on epilepsy as well as head nodding syndrome in Tanzania and her ever-lasting support, be it in the African fields or as an advisor to various projects via the internet. She has laid the foundation on which we are building our work of today on potential causes of epilepsy/head nodding syndrome; we are extremely grateful for her contribution.
Andrea Sylvia Winkler and Katrin Friedrich contributed equally to the conceptual work of the manuscript - and so did Thaddaeus Gotwald and Erich Schmutzhard

\section{References}

1. Winkler AS, Friedrich K, Konig R, Meindl M, Helbok R, Unterberger I, Gotwald T, Dharsee $\mathrm{J}$, et al. The head nodding syndrome-clinical classification and possible causes. Epilepsia 2008; 49:2008-2015.

2. Winkler AS, Friedrich K, Meindi M, Kidunda A, Nassri A, Jilek-Aall L, et al. Clinical characteristics of people with head nodding in Tanzania. Trop Doct 2010; 3:173-175.

3. Reik L, Abubakar A, Opoka M, Mindra G, Sejvar J, Dowell SF, et al. Centers for Disease Control and Prevention (CDC). Nodding syndrome - Southern Sudan, 2011. MMWR 2012; 61:52-54.

4. Tumwine JK, Vandemaele K, Chungong S, Richer M, Anker M, Ayana Y, et al. Clinical and epidemiologic characteristics of nodding syndrome in Mundri County, southern Sudan. Afr Health Sci. 2012 Sep;12(3):242-8.

5. Vogel G. Mysterious disease haunts region. Science 2012, 336:144-146.

6. Brattig NW. Pathogenesis and host responses in human onchocerciasis: impact of Onchocerca filariae and Wolbachia endobacteria. Microbes Infect 2004, 6:113--128.

7. Erttmann KD, Unnasch TR, Greene BM, Albiez EJ, Boateng J, Denke AM, et al. A DNA sequence specific for forest form Onchocerca volvulus. Nature 1987; 327:415--417.

8. Fuglsang H, Anderson J. Microfilariae of Onchocerca volvulus in blood and urine before, during, and after treatment with diethylcarbamazine. J Helminthol 1974; 48:93-97.

9. Duke BO, Vincelette J, Moore PJ. Microfilariae in the cerebrospinal fluid, and neurological complications, during treatment of onchocerciasis with diethylcarbamazine. Tropenmed Parasitol 1976; 27:123-1-32.

10. Kipp W, Kasoro S, Burnham G. Onchocerciasis and epilepsy in Uganda. Lancet 1994; 343:183184.

11. Jilek-Aall L: Neurofilariasis. Can Onchocerciasis cause Epilepsy? In Recent Advances in Tropical Neurology. Edited by Rose FC. Amsterdam: Elsevier Academic Press; 1995:283-288. 
12. Kaiser C, Kipp W, Asaba G, Mugisa C, Kabagambe G, Rating D, Leichsenring M. The prevalence of epilepsy follows the distribution of onchocerciasis in a west Ugandan focus. Bull World Health Organ 1996; 74:361-3-67.

13. Kaiser C, Asaba G, Leichsenring M, Kabagambe G. High incidence of epilepsy related to onchocerciasis in West Uganda. Epilepsy Res 1998; 30:247-251.

14. Newell ED, Vyungimana F, Bradley JE. Epilepsy, retarded growth and onchocerciasis, in two areas of different endemicity of onchocerciasis in Burundi. Trans R Soc Trop Med Hyg 1997; 91:525--527.

15. Boussinesq M, Pion SD, Demanga-Ngangue, Kamgno J. Relationship between onchocerciasis and epilepsy: a matched case-control study in the Mbam Valley, Republic of Cameroon. Trans R Soc Trop Med Hyg 2002; 96:537-541.

16. Kabore JK, Cabore JW, Melaku Z, DruetCabanac M, Preux PM. Epilepsy in a focus of onchocerciasis in Burkina Faso. Lancet 1996; 347:836.

17. Druet-Cabanac M, Preux PM, Bouteille B, Bernet-Bernady P, Dunand J, Hopkins A, et al. Onchocerciasis and epilepsy: a matched casecontrol study in the Central African Republic. Am J Epidemiol 1999; 149:565-570.

18. Farnarier G, Diop S, Coulibaly B, Arborio S, Dabo A, Diakite $M$, Traore $S$, et al. Onchocercose et épilepsie. Enquête épidémiologique au Mali. Med Trop (Mars) 2000; 60:151-155.

19. Druet-Cabanac M, Boussinesq M, Dongmo L, Farnarier G, Bouteille B, Preux PM. Review of epidemiological studies searching for a relationship between onchocerciasis and epilepsy. Neuroepidemiology 2004; 3:144-149.

20. Pion SD, Kaiser C, Boutros-Toni F, Cournil A, Taylor MM, Meredith SE, Stufe A, et al. Epilepsy in onchocerciasis endemic areas: systematic review and meta-analysis of population-based surveys. PLoS Negl Trop Dis 2009; 3:e461.

21. Marin B, Boussinesq M, Druet-Cabanac M, Kamgno J, Bouteille B, Preux PM. Onchocerciasis-related epilepsy? Prospects at a time of uncertainty. Trends Parasitol 2006; 22:1720.

22. Rwiza HT, Kilonzo GP, Haule J, Matuja WB, Mteza I, Mbena P, et al. Prevalence and incidence of epilepsy in Ulanga, a rural Tanzanian district:
A community-based study. Epilepsia 1992; 33:1051-1056.

23. Ngugi AK, Bottomley C, Kleinschmidt I, Wagner RG, Kakooza-Mwesige A, Ae-Ngibise $\mathrm{K}$, Owusu-Agyei S, et al. Prevalence of active convulsive epilepsy in sub-Saharan Africa andassociated risk factors: cross-sectional and case-control studies. Lancet Neurol 2013; 12:253263.

24. Mwaiko GL, Mtoi RS, Mkufya AR. Onchocerciasis prevalence in Tanzania. Cent Afr J Med 1990; 36:94-96.

25. Jilek-Aall L. Forty years of experience with epilepsy in Africa. In The Brainstorms Village Epilepsy in Our World. Edited by Schachter SC, Andermann LF. Philadelphia: Lippincott, Williams \& Wilkins; 2003:37-52.

26. Senanayake N, Roman G. Epidemiology of epilepsy in developing countries. Bull World Health Organ 1993; 71:247-258.

27. Zhang S, Li BW, Weil GJ. Paper Chromatography Hybridization: A rapid method for detection of onchocerca volvulus DNA amplified by PCR. Am J Trop Hyg 2000; 63:85-89.

28. International League Against Epilepsy, Commission on Neuroimaging. Recommendations for Neuroimaging of patients with epilepsy. Epilepsia 1997; 38:12551256.

29. Diop AG, De Boer HM, Mandlhate C, Prilipko L, Meinardi H. The global campaign against epilepsy in Africa. Act Trop 2003; 87:149-159.

30. Ogunniyi A, Adeyinka A, Fagbemi SO, Orere R, Falope ZF, Oyawole SO. Computerized tomographic findings in adolescent and adult Nigerian epileptics. West Afr J Med 1994; 13:128131.

31. Obajimi MO, Fatunde OJ, Ogunseyinde AO, Omigbodun OO, Atalabi OM, Joel RU. Computed tomography and childhood seizure disorder in Ibadan. West Afr J Med 2004; 23:16772.

32. Betting LE, Mory SB, Lopes-Cendes I, Li LM, Guerreiro MM, Guerreiro CAM, et al. MRI reveals structural abnormalities in patients with idiopathic generalized epilepsy. Neurology 2006; 67:848-852.

33. Brockhaus A, Elger CE. Complex partial seizures of temporal lobe origin in children of different age groups. Epilepsia 1995; 36:11731181. 
34. Matuja WBP. Aetiological factors in Tanzanian epileptics. East Afr Med J 1989; 66:343-348.

35. Matuja WBP, Kilonzo G, Mbena P, Mwango'mbola R, Wong P, Goodfellow P, et al. Risk factors for epilepsy in a rural Area in Tanzania: A community-based case-control study. Neuroepidemiology 2001; 20:242-247.

36. Edwards T, Scott AG, Munyoki G, Odera VM, Chengo E, Bauni E, Kwasa T, et al. Active convulsive epilepsy in a rural district of Kenya: a study of prevalence and possible risk factors. Lancet Neurol 2008; 7:50-56.

37. Yu R, Gao L, Mao B, He C, Zeng X. Gliosis: follow-up and pathological study of 34 cases. Zhonghua Wai Ke Za Zhi 1989; 36:469-471.

38. Kaiser C, Benninger C, Asaba G, Mugisa C, Kabagambe G, Kipp W, Rating D. Clinical and electro-clinical classification of epileptic seizures in West-Uganda. Bull Soc Pathol Exot 2000; 93:255-259.

39. Kobayashi E, Guerreiro CA, Cendes F. Late onset temporal lobe epilepsy with MRI evidence of mesial temporal sclerosis following acute neurocysticercosis: case report. Arq Neuropsiquiatr 2001; 59:255-258.

40. Lukiana T, Mandina M, Situakibanza NH, Mbula MM, Lepira BF, Odio WT, Kamgno J, Boussinesq M. A possible case of spontaneous Loa loa encephalopathy associated with a glomerulopathy. Filaria J 2006 ; 5:6.

41. Kamgno J, Boussinesq M, Labrousse F, Nkegoum B, Thylefors BI, Mackenzie CD. Case Report: Encephalopathy after Ivermectin Treatment in a Patient Infected with Loa Loa and Plasmodium spp. Am J Trop Med Hyg 2008; 78:546-551.

42. Helbok R, Brenneis C, Engelhardt K, Beer R, Lackner P, Brössner G, et al. A rare case of Toxocara canis cerebral vasculitis. Eur J Neurol 2007; 4:e49.

43. König R, Nassri A, Meindl M, Matuja W, Kidunda AR, Siegmund V, et al. The role of O.volvulus in the development of epilepsy in a rural area of Tanzania. Parasitology 2010; 137;1559-1568.

44. Gallin MY, Jacobi AB, Buttner DW, Schonberger O, Marti T, Erttmann KD. Human autoantibody to defensin: disease association with hyperreactive onchocerciasis (sowda). J Exp Med 1995; 182:41-47.
45. Ottesen EA. Immune responsiveness and the pathogenesis of human onchocerciasis. J Infect Dis 1995; 171:659-671.

46. McKechnie NM, Braun G, Connor V, Kläger S, Taylor DW, Alexander R A, Gilbert CE. Immunologic cross-reactivity in the pathogenesis of ocular onchocerciasis. Invest Ophthalmol Vis Sci 1993; 34:2888-2902.

47. Sejvar JJ, Kakooza AM, Foltz JL, Makumbi I, Atai-Omoruto AD, Malimbo M, Ndyomugyenyi R, et al. Clinical, neurological, and electrophysiological features of nodding syndrome in Kitgum, Uganda: an observational case series. Lancet Neurol 2013; 12:166-174. 